\title{
Glycine max Antigen IgE Antibody Measurement
}

National Cancer Institute

\section{Source}

National Cancer Institute. Glycine max Antigen IgE Antibody Measurement. NCI

Thesaurus. Code C147278.

The determination of the amount of Glycine max antigen IgE antibody present in a sample. 